\title{
The undergraduate premedical experience in the United States: a critical review
}

\author{
Katherine Y. Lin ${ }^{1}$, Sonali Parnami' ${ }^{2}$, Andrea Fuhrel-Forbis ${ }^{2}$, Renee R. Anspach ${ }^{1}$, Brett Crawford ${ }^{3}$, \\ Raymond G. De Vries ${ }^{2}$ \\ ${ }^{1}$ Department of Sociology, University of Michigan, Ann Arbor, MI, USA \\ ${ }^{2}$ Center for Bioethics and Social Sciences in Medicine, University of Michigan, Ann Arbor, MI, USA \\ ${ }^{3}$ School of Business Administration, Wayne State University, Detroit, MI, USA
}

Correspondence: Katherine Y. Lin, Department of Sociology, Literature Sciences and the Arts Building, Rm \#3001, $500 \mathrm{~S}$. State Street, Ann Arbor, MI, USA. Email: linkathy@umich.edu

\begin{abstract}
Objectives: To better understand the consequences of the premedical years for the character of (future) physicians by critically reviewing the empirical research done on the undergraduate premedical experience in the United States.

Methods: We searched ERIC, JSTOR, PubMed, Scopus, ISI Web of Science, and PsycINFO from the earliest available date for empirical, peer-reviewed studies of premedical students in the United States. We then used qualitative methods to uncover overall themes present in this literature. Results: The initial literature search identified 1,168 articles, 19 of which were included for review. Reviewed articles were published between 1976 and 2010 with the majority published prior to 1990. Articles covered two broad topics:
\end{abstract}

explaining attrition from the premedical track, and investigating the personality traits and stereotypes of premedical students. Self-selection bias and high attrition rates were among the limitations of the reviewed articles.

Conclusions: There is very little current research on the premedical experience. Given the importance of the premedical years on the process of becoming a medical professional, it is imperative that we do more and better research on how the premedical experience shapes future physicians. Keywords: Premedical education, premedical syndrome, attrition from premedical track, hidden curriculum, professionalization

\section{Introduction}

Scholars and policy makers have long been concerned with the selection and training of the future physician workforce--concerned not only with the academic competence of future doctors, but also with the quality of their character, including their ability to reason morally, to listen to patients, and to empathize with patients as whole persons, rather than seeing them as collections of genes, cells, and organs. While prior research in this area has largely considered the influences of the criteria for admission into medical school or how students are influenced by their medical training, ${ }^{1}$ more recently, scholars have begun to consider how the premedical years shape future physicians. $^{2-4}$ One indicator of this new interest in the premedical years is the recent decision by the Association of American Medical Colleges to revise the Medical College
Admissions Test (MCAT) to include questions on the social and behavioral sciences so as to encourage premedical students to study these topics. ${ }^{5}$

Empirical evidence has demonstrated that what happens to students prior to entering medical school affects their performance during medical school and beyond. For instance, there are documented correlations between premedical academic performance and pre-clinical academic performance in the UK, ${ }^{6,7}$ the US, ${ }^{8}$ and elsewhere. ${ }^{9}$ Others have argued that students enter medical school with already formed values and ethical points of view that may be difficult to influence or alter with current bioethics curricula in medical schools. ${ }^{10,11}$ Recent studies on physician depression and burnout also indicate that physician wellbeing is diminished by the stress of medical and premedical education. ${ }^{12-14}$ These studies and policy changes suggest that 
the premedical years have a significant influence on the character and well-being of physicians.

Most prior research on the premedical years has focused on how the academic experience - in particular, the performance in, and choice of, coursework during these years influences outcomes during medical school years and beyond. ${ }^{15}$ While important, the academic experience is only one aspect of the premedical years. Premedical education, like medical education, includes the formal curriculum, as well as informal and hidden curricula. ${ }^{16}$ The entire experience of the premedical years - including academic and formal curricular training as well as informal and cocurricular experiences, such as cooperating with and competing against classmates in required coursework, participating in a variety of extracurricular activities, and repeatedly examining their ambitions to become doctors influences future physicians. Although there have been several reviews of how the academic performance of premedical students influences their academic performance in medical school and in practice, ${ }^{17,18}$ to our knowledge no similar synthesis of the literature on other aspects of the premedical experience has been done.

Our goal in this review is to critically synthesize what is known about the premedical experience in the United States in order to generate research questions for future scholarship. ${ }^{19}$ We chose to study premedical students in the United States because in the US the premedical years are well defined, offering a focused look at the experience of "being a premed". What we learn here may be compared to other medical education systems where the "premedical years" occur in secondary school. In the US context, we reviewed studies of students in four-year post-secondary institutions. There are other avenues for gaining admission to American medical schools, but the vast majority of those who apply to US medical schools graduate from this type of institution. ${ }^{20}$

\section{Defining the premedical experience}

Though there is no single or standard pathway into medical school in the United States, the simple act of planning to go to medical school subjects a student to a particular set of requirements and competitive pressures. The curricular requirements and extra-curricular expectations for gaining admission to medical school-established by individual medical schools and by the content areas of the MCAT provide the overall structure of the premedical years. The premedical experience, however, encompasses all of the things students do inside and outside the classroom strategizing, competing, and collaborating- to successfully master challenging academic material and satisfactorily meet requirements in hopes of putting together a successful medical school application. Thus, the premedical experience is determined by far more than one's academic skill or personality - it is the informal, yet patterned and collective response to the requirements of securing a career as a physician.

In the United States, for instance, students must not only score well on the MCAT, but they must also do well in large "weeder" courses (most notably, organic chemistry, a course that separates the "smart" from the "not-so-smart" students) while carefully crafting a résumé of desirable extra-curricular activities, and balancing academic, social, and personal lives. Students in countries where admission to medical school occurs directly after secondary education face similar requirements. For example, students in the United Kingdom must take the required number of A-level courses in a variety of subjects, do well on an Aptitude Test (e.g. the UKCAT: UK Clinical Aptitude Test), and enhance their attractiveness to medical schools with participation in extracurricular activities. These requirements amount to a type of cultural pressure that contributes to the socialization of future physicians. Thus, the character of the next generation of physicians is forged long before students walk through the doors of medical school: medical socialization begins with the negotiation of the premedical years.

Participants in the premedical experience include not only those who will become medical students and practicing physicians, but also those who initially express interest in medical studies but end up pursuing other careers. Premedical students who choose not to apply to medical school are also crucial participants in the premedical experience, and also help shape the competitive pressures and collective experiences of all premedical students. Thus, studies that examine the premedical years by asking only medical students (i.e., those who have successfully gained entry to medical school) miss an essential part of the premedical experience. Similarly, studies that use data from students in special pipeline programs (ie. B.A/MD programs) may also not capture the full extent of the premedical experience since these students are subject to a different set of environmental pressures. In fact, many of these programs were established initially to combat competitive pressures present in the modal premedical experience. ${ }^{21}$ Students in pipeline programs may have stronger ties to medical school upon matriculation into these programs, increased academic and support for medical studies, and face different competitive pressures as many programs do not require these students to take the MCAT for admission. ${ }^{21}$ For this reason, to accurately capture the premedical experience, we review studies of premedical students, excluding studies of students in pipeline programs, during their premedical years.

\section{Methods}

We began our review by searching through databases relevant to medical education and social science research: ERIC, JSTOR, PubMed, Scopus, ISI Web of Science, and PsycINFO. Using intentionally broad search terms, we included appropriate combinations of keywords and 
controlled vocabulary terms relating to premedical education (See Appendix I: Databases searched \& search strategies). No limits were placed on date, language or article type during the retrieval process (done on June 16, 2010). To ensure we did not miss a study that met our criteria, we also searched the references of all full-review articles for relevant studies.

The first author reviewed the 1,168 titles generated from the initial search and selected 574 relevant articles for abstract review (See Figure 1). Articles were discarded if the titles indicated the sample were not United States premedical students. The majority of the articles discarded used the term "premedical" or "undergraduate medical education" to refer to the first two years of formal medical training before clinical rotations rather than undergraduate education leading to a bachelor's degree. Articles were included for abstract review if it was not clear from the title that the research question dealt with premedical students in the United States. The first and second authors reviewed abstracts from each of the articles to select articles for full text review; the third author was consulted to resolve disagreements. Persisting disagreements were resolved by discussion with all authors. The first and second authors then independently reviewed fifty-eight full-text articles to determine final inclusion (with the third author resolving disagreements and persisting disagreements resolved via group discussion). Nineteen articles met inclusion criteria for this review.

Our main selection criterion was that the study needed to have sampled premedical students during their premedical years (see Table 1). Given our focus on all premedical students (and not just those who succeeded in gaining admission to medical school) we excluded studies that sampled only medical students to draw conclusions about the premedical experience. Additionally, all articles examining premedical students in the US published in peerreviewed journals were considered for inclusion, while monographs, book reviews, national reports, and conference proceedings were excluded. Because we are interested in learning about the premedical experience and not just premedical coursework, we excluded studies that did not gather primary data from premedical students - such as studies that examined only undergraduate students' academic transcripts. We also excluded studies that exclusively examined students from combined BA/MD programs, post-baccalaureate programs, and special medical pipeline programs, since students in these programs already have guaranteed admission into medical school at the undergraduate level, or follow pathways into medical school that are unconventional, and therefore do not reflect the modal experience of premedical students.

The first author, assisted by the second author, summarized all articles and developed a typology of the overarching themes found in the literature. Themes were then grouped together into broader analytic questions that have been asked about the premedical experience. Since the study did not directly collect data from human subjects, research ethics approval was not obtained.

\section{Results}

The nineteen articles we reviewed were published between 1976 and 2010 and varied greatly in focus and design (Table 2). The articles were methodologically diverse: twelve articles were based on large sample surveys of premedical students; five were based on qualitative interviews with smaller samples of premedical students, and two used a mixed-method approach. This empirical work on premedical students can be categorized into two broad research agendas: explaining attrition from the premedical track, and investigating the personality traits and stereotypes of premedical students.

Table 1. Inclusion and exclusion criteria for study

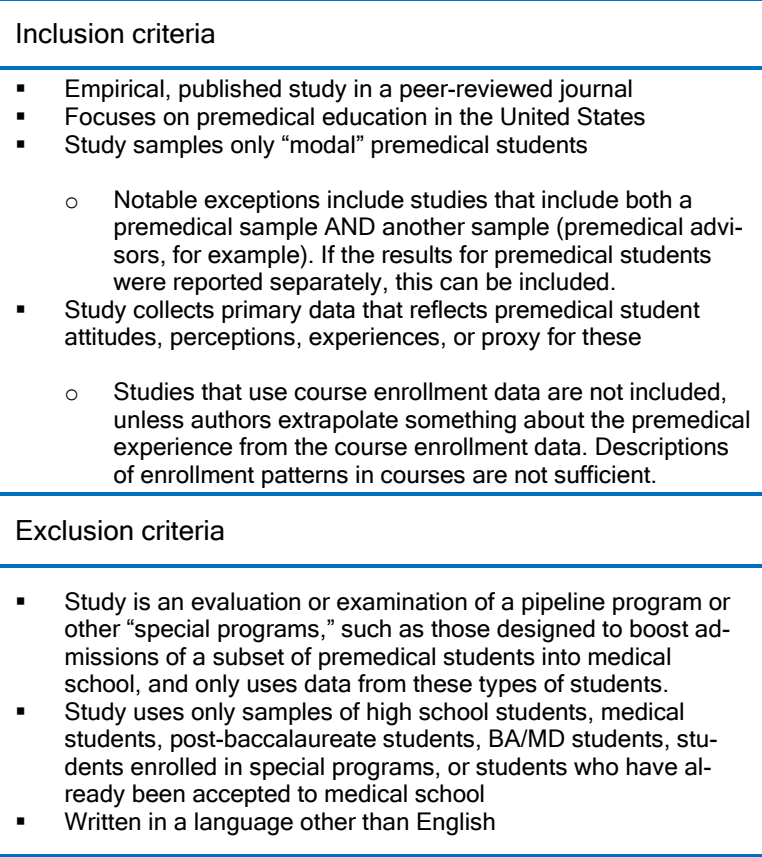

\section{Attrition from the premedical track}

Eleven of the nineteen articles reviewed attempted to explain why certain students entered their undergraduate studies with an interest in medicine but ended up pursuing other aspirations. These articles focused on why women and under-represented minorities disproportionately leave the premedical track. The earlier work in this group, done between the 1970s through the 1990s, found that from the beginning of their premedical years women were less certain than men about their medical career aspirations, ${ }^{22}$ or had less of a normative disincentive to abandon their original aspirations. ${ }^{23,24}$ Other work from the early 1990s found that premedical students, in general, decided not to apply to medical school either because they were no longer interested in medicine or because they realized that their previous expectations of what it would be like to be a physician no longer matched what they now viewed as the 


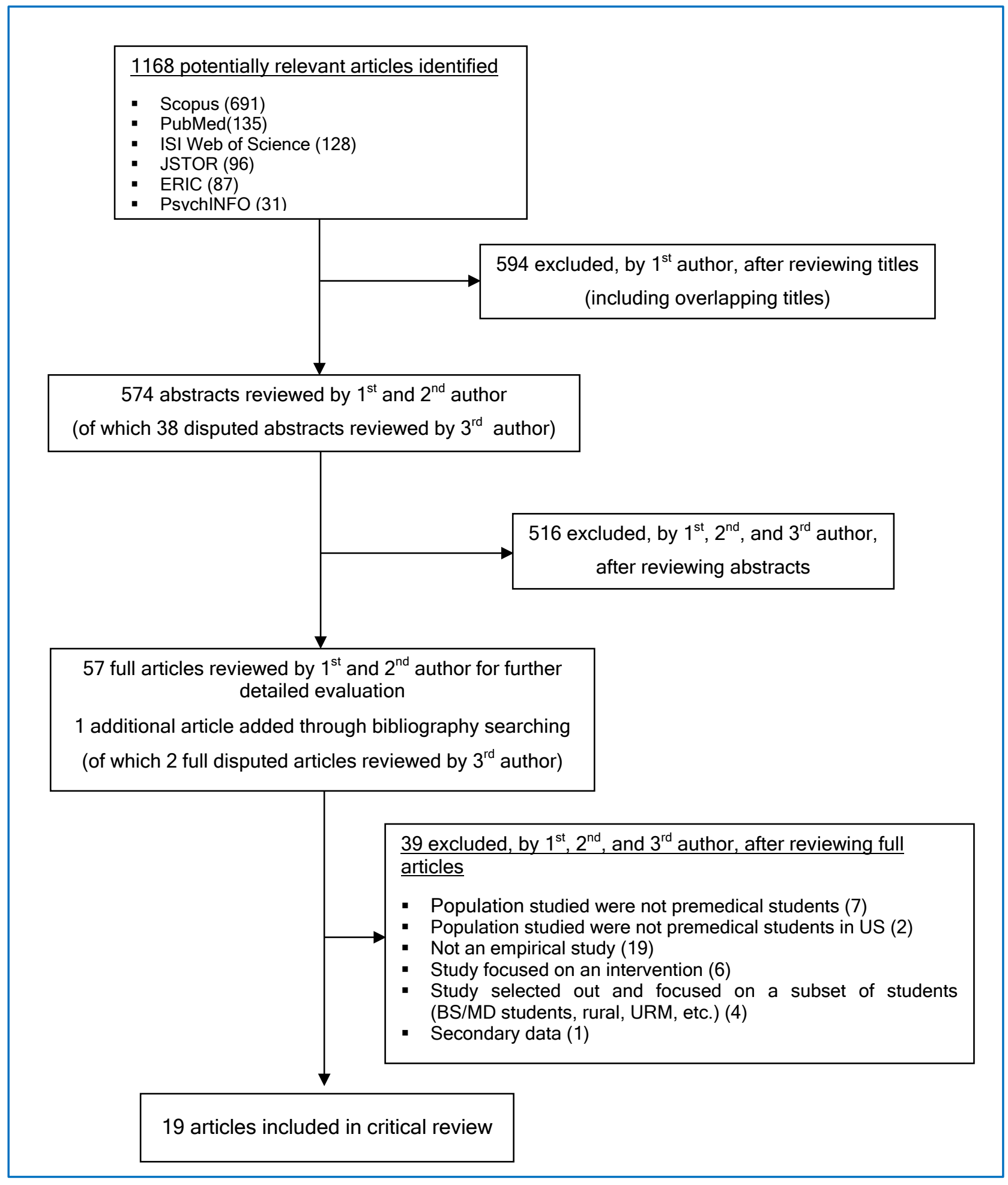

Figure 1. Selection of the articles for the critical review 
Lin et al. The premedical experience

Table 2. Studies included in critical review

\begin{tabular}{|c|c|c|c|c|c|c|c|}
\hline Source & Research question & Method & Measures & Population & Sample size & $\begin{array}{l}\text { Response } \\
\text { rate }\end{array}$ & $\begin{array}{l}\text { Methodological } \\
\text { limitations }\end{array}$ \\
\hline $\begin{array}{l}\text { Barondess } \\
\text { and Glaser } \\
1993^{26}\end{array}$ & $\begin{array}{l}\text { What are the attitudes } \\
\text { that lead potential } \\
\text { medical school } \\
\text { applicants towards or } \\
\text { away from medical } \\
\text { school? }\end{array}$ & $\begin{array}{l}\text { Survey / } \\
\text { Cross- } \\
\text { sectional }\end{array}$ & $\begin{array}{l}\text { Attitudes towards } \\
\text { medical careers-- } \\
\text { such importance of } \\
\text { job satisfaction, } \\
\text { stability of career, } \\
\text { and ability to help } \\
\text { those who are less } \\
\text { fortunate in } \\
\text { choosing a medical } \\
\text { career }\end{array}$ & $\begin{array}{l}\text { National sample of } \\
\text { college students } \\
\text { and premedical } \\
\text { advisors from } 100 \\
\text { US schools }\end{array}$ & $\begin{array}{l}\text { Study 1: total sample } \\
1780 \text { students (1003 } \\
\text { non-applicants to } \\
\text { medical school; } 500 \\
\text { medical school } \\
\text { applicants; } 227 \\
\text { qualified non- } \\
\text { applicants); Study } \\
\text { 2:93 premedical } \\
\text { advisors }\end{array}$ & $\begin{array}{l}\text { Study 1: } \\
\text { Student } \\
\text { groups: } 84- \\
\text { 89\%; Study 2: } \\
\text { Premedical } \\
\text { advisors: 93\% }\end{array}$ & $\begin{array}{l}\text { Limited infor- } \\
\text { mation provided } \\
\text { regarding interview } \\
\text { items or whether } \\
\text { items were } \\
\text { pretested. }\end{array}$ \\
\hline $\begin{array}{l}\text { Barr et al } \\
2008^{27}\end{array}$ & $\begin{array}{l}\text { What are the causes, } \\
\text { among underrepre- } \\
\text { sented minority } \\
\text { students, of a decline } \\
\text { in interest in } \\
\text { pursuing a career in } \\
\text { medicine? }\end{array}$ & $\begin{array}{l}\text { Study 1: } \\
\text { Survey/ } \\
\text { Longitudinal; } \\
\text { Study 2: } \\
\text { Follow-up } \\
\text { interviews } \\
\text { with subset of } \\
\text { Study } 1 \text { survey } \\
\text { participants }\end{array}$ & $\begin{array}{l}\text { Survey measure: } \\
\text { 10-point scale of } \\
\text { interest in } \\
\text { premedical studies. } \\
\text { Interviews asked } \\
\text { about factors } \\
\text { leading to a change } \\
\text { in interest, the role } \\
\text { of coursework in } \\
\text { changing interests, } \\
\text { and important } \\
\text { skills or resources } \\
\text { premedical } \\
\text { students need to } \\
\text { succeed. }\end{array}$ & $\begin{array}{l}\text { Incoming } \\
\text { freshman college } \\
\text { students who } \\
\text { indicated interest } \\
\text { in medicine and } \\
\text { completed all } 3 \\
\text { surveys, Stanford } \\
\text { University }\end{array}$ & $\begin{array}{l}\text { Study 1: } 362 \text { students; } \\
\text { Study } \\
\text { 2:68 students }\end{array}$ & $\begin{array}{l}\text { Study 1: } \\
\text { Initial } \\
\text { response rate } \\
\text { to survey } \\
\text { invitation not } \\
\text { reported; } \\
34.3 \% \text { of } 1056 \\
\text { T1 respond- } \\
\text { ents } \\
\text { completed all } \\
3 \text { surveys; } \\
\text { Study 2: Not } \\
\text { reported }\end{array}$ & $\begin{array}{l}\text { Limited generali- } \\
\text { zability - sampled } \\
\text { only from one } \\
\text { university, which is } \\
\text { among the most } \\
\text { selective nationally; } \\
\text { Students self- } \\
\text { selected to take the } \\
\text { survey; High rate of } \\
\text { attrition over time }\end{array}$ \\
\hline $\begin{array}{l}\text { Barr et al } \\
2010^{28}\end{array}$ & $\begin{array}{l}\text { Do negative } \\
\text { experiences in } \\
\text { chemistry courses } \\
\text { contribute to } \\
\text { undergraduate } \\
\text { students discontinu- } \\
\text { ing premedical } \\
\text { studies? }\end{array}$ & $\begin{array}{l}\text { Study 1: } \\
\text { Sur- } \\
\text { vey/Repeated } \\
\text { Measures; } \\
\text { Study 2: } \\
\text { Follow-up } \\
\text { interviews } \\
\text { with subset of } \\
\text { survey } \\
\text { participants }\end{array}$ & $\begin{array}{l}\text { Survey measure: } \\
10 \text {-point scale of } \\
\text { interest in } \\
\text { premedical studies. } \\
\text { Interviews asked } \\
\text { about factors } \\
\text { leading to a change } \\
\text { in interest, and to } \\
\text { identify courses } \\
\text { that discouraged } \\
\text { interest. }\end{array}$ & $\begin{array}{l}\text { Incoming } \\
\text { freshman college } \\
\text { students who } \\
\text { indicated an } \\
\text { interest in } \\
\text { medicine, UC } \\
\text { Berkeley }\end{array}$ & $\begin{array}{l}\text { Study 1: } 1036 \\
\text { students; Study 2:63 } \\
\text { students completed } \\
\text { follow-up interviews }\end{array}$ & $\begin{array}{l}\text { Study 1: } \\
\text { Initial } \\
\text { response rate } \\
\text { to survey } \\
\text { invitation not } \\
\text { reported; } 57 \% \\
\text { of the } 1036 \mathrm{~T} 1 \\
\text { respondents } \\
\text { also respond- } \\
\text { ed at the end } \\
\text { of their } \\
\text { second year; } \\
\text { Study 2: Not } \\
\text { reported }\end{array}$ & $\begin{array}{l}\text { Limited generali- } \\
\text { zability- sampled } \\
\text { only from one } \\
\text { university, which is } \\
\text { among the most } \\
\text { selective national- } \\
\text { ly; Students self- } \\
\text { selected to take the } \\
\text { survey; Initial } \\
\text { response rate not } \\
\text { reported; High rate } \\
\text { of attrition over } \\
\text { time; Students not } \\
\text { individually } \\
\text { identified so } \\
\text { unable to track } \\
\text { individual change } \\
\text { over time }\end{array}$ \\
\hline $\begin{array}{l}\text { Chuck } \\
1996^{32}\end{array}$ & $\begin{array}{l}\text { What are premedical } \\
\text { students' expectations } \\
\text { from a medical } \\
\text { career? }\end{array}$ & $\begin{array}{l}\text { Survey/ } \\
\text { Cross- } \\
\text { sectional }\end{array}$ & $\begin{array}{l}\text { Expectations of a } \\
\text { medical career } \\
\text { such as the ability } \\
\text { of physicians to } \\
\text { cure and help their } \\
\text { patients, to have } \\
\text { intellectually } \\
\text { satisfying work, or } \\
\text { to have to perform } \\
\text { administrative } \\
\text { duties }\end{array}$ & $\begin{array}{l}\text { Study 1: premedi- } \\
\text { cal students, UC } \\
\text { Berkeley and Cal } \\
\text { State Hayward; } \\
\text { Study 2; practicing } \\
\text { physicians from } \\
\text { similar geographic } \\
\text { area }\end{array}$ & $\begin{array}{l}\text { Study 1:84 premedi- } \\
\text { cal students; Study } \\
\text { 2:251 practicing } \\
\text { physicians }\end{array}$ & $\begin{array}{l}\text { Study 1: } \\
\text { premedical } \\
\text { students: } \\
\text { 100\%; Study } \\
\text { 2: physicians: } \\
62 \%\end{array}$ & $\begin{array}{l}\text { Possible limited } \\
\text { generalizability } \\
\text { because this sample } \\
\text { of premedical } \\
\text { students may be } \\
\text { more engaged than } \\
\text { the general } \\
\text { population of } \\
\text { premedical } \\
\text { students; No } \\
\text { statistical tests of } \\
\text { magnitude of } \\
\text { between-subjects } \\
\text { comparisons }\end{array}$ \\
\hline $\begin{array}{l}\text { Colquitt } \\
\text { and Killian } \\
1991^{25}\end{array}$ & $\begin{array}{l}\text { Why do students who } \\
\text { take the MCAT opt to } \\
\text { not apply to medical } \\
\text { school in the years } \\
1986 \text { and } 1988 ?\end{array}$ & $\begin{array}{l}\text { Study 1: } \\
\text { Secondary } \\
\text { data analysis; } \\
\text { Studies } 2 \& 3 \text { : } \\
\text { Survey/ cross- } \\
\text { sectional with } \\
\text { open ended } \\
\text { response }\end{array}$ & $\begin{array}{l}\text { Reasons for not } \\
\text { applying to } \\
\text { medical school, } \\
\text { attitudes about } \\
\text { medical practice }\end{array}$ & $\begin{array}{l}\text { Study 1: MCAT } \\
\text { examinees; Studies } \\
2 \text { \& 3: non- } \\
\text { applicants to } \\
\text { medical school } \\
\text { (took MCAT but } \\
\text { did not apply) }\end{array}$ & $\begin{array}{l}\text { Study 1: secondary } \\
\text { data analysis (n/a); } \\
\text { Study 2: } 539 \text { non- } \\
\text { applicants (1986); } \\
\text { Study 3: } 745 \text { non- } \\
\text { applicants (1988) }\end{array}$ & $\begin{array}{l}\text { Study 1: } \\
\text { secondary } \\
\text { data analysis } \\
\text { (n/a); Study 2: } \\
\text { 35\% (1986); } \\
\text { Study 3: 32\% } \\
\text { (1988) }\end{array}$ & $\begin{array}{l}\text { Self-selection bias } \\
\text { possible due to low } \\
\text { response rate } \\
\text { (authors used } \\
\text { weighted analysis } \\
\text { to correct for non- } \\
\text { response bias) }\end{array}$ \\
\hline
\end{tabular}


Table 2. Studies included in critical review (cont)

\begin{tabular}{|c|c|c|c|c|c|c|c|}
\hline Source & Research question & Method & Measures & Population & Sample size & $\begin{array}{l}\text { Response } \\
\text { rate }\end{array}$ & $\begin{array}{l}\text { Methodological } \\
\text { limitations }\end{array}$ \\
\hline $\begin{array}{l}\text { Conrad } \\
1986^{40}\end{array}$ & $\begin{array}{l}\text { How prevalent is the } \\
\text { premedical cut-throat } \\
\text { stereotype in reality? } \\
\text { Despite a low } \\
\text { prevalence, why does } \\
\text { the myth still exist? }\end{array}$ & $\begin{array}{l}\text { Interviews/ } \\
\text { Cross- } \\
\text { sectional }\end{array}$ & $\begin{array}{l}\text { Asked interviewees } \\
\text { about the } \\
\text { premedical sub- } \\
\text { culture, and } \\
\text { conducted } \\
\text { fieldwork in } \\
\text { premedical settings }\end{array}$ & $\begin{array}{l}\text { Premedical } \\
\text { students, } \\
\text { Brandeis } \\
\text { University }\end{array}$ & $\begin{array}{l}30 \text { premedical } \\
\text { students }\end{array}$ & $\begin{array}{l}\text { Potential } \\
\text { participants } \\
\text { approached- } \\
\text { not reported }\end{array}$ & $\begin{array}{l}\text { Half of sample was } \\
\text { convenience sample; } \\
\text { Limited generalizabil- } \\
\text { ity to other institu- } \\
\text { tions due to selective, } \\
\text { private nature, small } \\
\text { institutional size, and } \\
\text { high focus on } \\
\text { premedical program } \\
\text { at Brandeis }\end{array}$ \\
\hline $\begin{array}{l}\text { Fiorentine } \\
1987^{23}\end{array}$ & $\begin{array}{l}\text { What causes the } \\
\text { premedical persis- } \\
\text { tence gap? Why do } \\
\text { more men persist in } \\
\text { premedical studies } \\
\text { than women? }\end{array}$ & $\begin{array}{l}\text { Study 1: } \\
\text { Secondary } \\
\text { data analysis; } \\
\text { Study 2: } \\
\text { Interviews/ } \\
\text { Cross- } \\
\text { sectional }\end{array}$ & $\begin{array}{l}\text { Student academic } \\
\text { achievement, } \\
\text { persistence } \\
\text { (continuing with } \\
\text { premedical studies, } \\
\text { application to } \\
\text { medical school), } \\
\text { interviews focus on } \\
\text { why students want } \\
\text { to become doctors }\end{array}$ & $\begin{array}{l}\text { Study 1: } \\
\text { academic } \\
\text { transcripts of } \\
\text { likely premedical } \\
\text { students; Study } \\
2 \text { : interviews } \\
\text { with premedical } \\
\text { students (GPA } \\
2.50-3.50 \text { ), } \\
\text { SUNY Stony } \\
\text { Brook }\end{array}$ & $\begin{array}{l}\text { Study 1: total sample } \\
673 \text { students ( } 323 \\
\text { females, } 350 \text { males); } \\
\text { Study } 2 \text { : total sample } \\
27 \text { students ( } 16 \\
\text { females, } 11 \text { males) }\end{array}$ & $\begin{array}{l}\text { Study 1: n/a; } \\
\text { Study 2: } \\
\text { Potential } \\
\text { participants } \\
\text { approached - } \\
\text { not reported }\end{array}$ & $\begin{array}{l}\text { "Premedical" as } \\
\text { defined by author } \\
\text { could include } \\
\text { students who were } \\
\text { not actually } \\
\text { premedical; limited } \\
\text { detail given of } \\
\text { development of } \\
\text { interview protocol or } \\
\text { selection of interview } \\
\text { participants; Study 2: } \\
\text { Limited generalizabil- } \\
\text { ity due to small } \\
\text { sample size for } \\
\text { interviews; Inferences } \\
\text { and conclusions } \\
\text { made from interview } \\
\text { responses reach } \\
\text { beyond scope of data }\end{array}$ \\
\hline $\begin{array}{l}\text { Fiorentine } \\
\text { and Cole } \\
1992^{24}\end{array}$ & $\begin{array}{l}\text { Why do fewer women } \\
\text { apply to medical } \\
\text { school if they are as } \\
\text { likely as men to get } \\
\text { into and through } \\
\text { medical school? }\end{array}$ & $\begin{array}{l}\text { Study 1: } \\
\text { Interviews/ } \\
\text { Cross- } \\
\text { sectional; } \\
\text { Study 2: } \\
\text { Interviews } \\
\text { with subset } \\
\text { from Study 1; } \\
\text { Study 3: } \\
\text { Interviews/ } \\
\text { Cross- } \\
\text { sectional with } \\
\text { separate } \\
\text { population }\end{array}$ & $\begin{array}{l}\text { Student academic } \\
\text { achievement } \\
\text { (transcripts) and } \\
\text { application to } \\
\text { medical school } \\
\text { status, self- } \\
\text { reported measures } \\
\text { of persistence, level } \\
\text { of encouragement } \\
\text { perceived, and } \\
\text { family formation } \\
\text { plans }\end{array}$ & $\begin{array}{l}\text { Study 1: } 542 \\
\text { persisting and } \\
\text { defecting } \\
\text { premedical } \\
\text { students } \\
\text { currently } \\
\text { enrolled at time } \\
\text { of study, SUNY } \\
\text { Stony Brook; } \\
\text { Study 2: } 36 \\
\text { premedical } \\
\text { students; Study } \\
\text { 3: } 62 \text { high school } \\
\text { students with } \\
\text { premedical plans }\end{array}$ & $\begin{array}{l}\text { Study 1: total sample } \\
542 \text { students ( } 240 \\
\text { females, } 302 \text { males); } \\
\text { Study 2: total sample } \\
36 \text { students ( } 23 \\
\text { female, } 13 \text { male); } \\
\text { Study 3: } 62 \text { students }\end{array}$ & $\begin{array}{l}\text { Study 1: } 542 \\
\text { of } 543(99.8 \%) \\
\text { students } \\
\text { participated in } \\
\text { telephone } \\
\text { interview; } \\
\text { Study 2: } \\
\text { Potential } \\
\text { participants } \\
\text { approached - } \\
\text { not reported; } \\
\text { Study 3: } 62 \text { of } \\
62 \text { (100\%) } \\
\text { eligible } \\
\text { interviews } \\
\text { completed }\end{array}$ & $\begin{array}{l}\text { Limited detail given } \\
\text { of development of } \\
\text { interview protocol or } \\
\text { selection of interview } \\
\text { participants; Selective } \\
\text { attrition due to } \\
\text { defection from } \\
\text { premedical program; } \\
\text { Lack of transparency } \\
\text { in reporting results } \\
\text { (study samples } \\
\text { grouped) }\end{array}$ \\
\hline $\begin{array}{l}\text { Hackman et } \\
\text { al } 197933\end{array}$ & $\begin{array}{l}\text { What are the } \\
\text { perceptions of } \\
\text { premedical students } \\
\text { held by both } \\
\text { premedical and non- } \\
\text { premedical students? }\end{array}$ & $\begin{array}{l}\text { Survey/ } \\
\text { Cross- } \\
\text { sectional with } \\
\text { closed and } \\
\text { open-ended } \\
\text { responses }\end{array}$ & $\begin{array}{l}\text { Ratings of students' } \\
\text { feelings towards } \\
\text { other student } \\
\text { groups (ie. Premed, } \\
\text { pre-law, etc) }\end{array}$ & $\begin{array}{l}\text { Undergraduate } \\
\text { college students, } \\
\text { Yale }\end{array}$ & $\begin{array}{l}\text { Total sample } 317 \\
\text { students (132 } \\
\text { sophomores and } 106 \\
\text { seniors randomly } \\
\text { selected, } 79 \text { seniors } \\
\text { who applied to } \\
\text { medical school) }\end{array}$ & $65.40 \%$ & $\begin{array}{l}\text { Limited generalizabil- } \\
\text { ity - sampled from } \\
\text { one private selective } \\
\text { university. }\end{array}$ \\
\hline $\begin{array}{l}\text { Horowitz } \\
201039\end{array}$ & $\begin{array}{l}\text { What achievement } \\
\text { goal orientations are } \\
\text { adopted by premedi- } \\
\text { cal students? How } \\
\text { does this differ by } \\
\text { subject, major, } \\
\text { requirements? }\end{array}$ & $\begin{array}{l}\text { Semi- } \\
\text { structured } \\
\text { interviews }\end{array}$ & $\begin{array}{l}\text { Main interview } \\
\text { question: "In which } \\
\text { subject areas do } \\
\text { you choose courses } \\
\text { just to get an easy } \\
\text { A and in which } \\
\text { subject areas do } \\
\text { you choose more } \\
\text { challenging courses } \\
\text { because of an } \\
\text { interest or a desire } \\
\text { to learn?" }\end{array}$ & $\begin{array}{l}\text { Undergraduate } \\
\text { college students } \\
\text { who completed } \\
\text { Organic } \\
\text { chemistry } \\
\text { during 2006-07 } \\
\text { year at a small, } \\
\text { all-male, liberal } \\
\text { arts college } \\
\text { contained within } \\
\text { a private Jewish } \\
\text { university in the } \\
\text { northeastern } \\
\text { U.S. }\end{array}$ & $\begin{array}{l}\text { Total sample } 30 \\
\text { students ( } 87 \% \\
\text { premedical; } 84 \% \\
\text { majoring in biology } \\
\text { or chemistry. }\end{array}$ & $\begin{array}{l}31(54.4 \%) \text { of } \\
\text { the } 57 \\
\text { students } \\
\text { completing } \\
\text { organic } \\
\text { chemistry } \\
\text { agreed to } \\
\text { participate - } \\
\text { data from } 30 \\
\text { interviews } \\
\text { reported }\end{array}$ & $\begin{array}{l}\text { Limited generalizabil- } \\
\text { ity - low response } \\
\text { rate, sampled from } \\
\text { one small, all-male, } \\
\text { private, northeastern } \\
\text { US, Jewish university }\end{array}$ \\
\hline
\end{tabular}


Lin et al. The premedical experience

Table 2. Studies included in critical review (cont)

\begin{tabular}{|c|c|c|c|c|c|c|c|}
\hline Source & Research question & Method & Measures & Population & Sample size & $\begin{array}{l}\text { Response } \\
\text { rate }\end{array}$ & $\begin{array}{l}\text { Methodological } \\
\text { limitations }\end{array}$ \\
\hline $\begin{array}{l}\text { Klink et al } \\
2008^{31}\end{array}$ & $\begin{array}{l}\text { Is there a relationship } \\
\text { between premedical } \\
\text { and non-premedical } \\
\text { students' perceived } \\
\text { level of family } \\
\text { support and } \\
\text { confidence in their } \\
\text { abilities to cope with } \\
\text { premedical life? }\end{array}$ & $\begin{array}{l}\text { Survey/ } \\
\text { Cross- } \\
\text { sectional }\end{array}$ & $\begin{array}{l}\text { Scale of coping } \\
\text { efficacy, self- } \\
\text { reported measures } \\
\text { of family support }\end{array}$ & $\begin{array}{l}\text { Premedical, } \\
\text { University of } \\
\text { Wisconsin. }\end{array}$ & $\begin{array}{l}\text { Total sample } 238 \\
\text { students }\end{array}$ & $\begin{array}{l}57 \% \text { of } \\
\text { expected } \\
\text { estimate of } \\
\text { participants } \\
\text { responded }\end{array}$ & $\begin{array}{l}\text { Limited generalizability } \\
\text { - low response rate, } \\
\text { sampled from one } \\
\text { large, competitive, } \\
\text { Midwestern university; } \\
\text { Study used limited } \\
\text { dimensions of family } \\
\text { support which may be } \\
\text { related to coping } \\
\text { efficacy. }\end{array}$ \\
\hline
\end{tabular}

\begin{tabular}{|c|c|c|c|c|c|c|c|}
\hline $\begin{array}{l}\text { Lewis } \\
1985^{38}\end{array}$ & $\begin{array}{l}\text { Does the career } \\
\text { choice of medicine } \\
\text { influence a premedi- } \\
\text { cal student to } \\
\text { undertake certain } \\
\text { types of coursework } \\
\text { at a liberal arts } \\
\text { college? }\end{array}$ & $\begin{array}{l}\text { Study 1: } \\
\text { Survey/ } \\
\text { Cross- } \\
\text { sectional; } \\
\text { Study 2: } \\
\text { Sur- } \\
\text { vey/Longitudi } \\
\text { nal of subset } \\
\text { from Study } 1 .\end{array}$ & $\begin{array}{l}\text { Self-reported } \\
\text { measures of career } \\
\text { goals, degree goals, } \\
\text { college major, } \\
\text { personality traits, } \\
\text { and interactional } \\
\text { styles }\end{array}$ & $\begin{array}{l}\text { Study 1: } \\
\text { Premedical and } \\
\text { non-premedical } \\
\text { students in three } \\
\text { classes } 1982 \text {, } \\
1983,1985, \\
\text { Macalester } \\
\text { College, St. Olaf } \\
\text { College; Study 2: } \\
\text { Classes 1983 and } \\
1985 \text { resurveyed. }\end{array}$ & $\begin{array}{l}\text { Study 1: Total sample } \\
345 \text { students ( } 58 \\
\text { premedical, } 99 \\
\text { biology/chem majors, } \\
187 \text { others); Study 2: } \\
\text { Not reported. }\end{array}$ & $\begin{array}{l}\text { Study } 1 \& \\
\text { Study 2: } \\
\text { Potential } \\
\text { participants } \\
\text { approached - } \\
\text { not reported }\end{array}$ & $\begin{array}{l}\text { Study } 1 \text { \& 2: Limited } \\
\text { generalizability- } \\
\text { sampled from two } \\
\text { small, liberal arts } \\
\text { colleges; Data presents } \\
\text { limited means of } \\
\text { testing study hypothe- } \\
\text { ses; Study 2: No } \\
\text { population data or } \\
\text { report on loss to } \\
\text { follow-up. }\end{array}$ \\
\hline $\begin{array}{l}\text { Lovecchio } \\
\text { and } \\
\text { Dundee } \\
2002^{29}\end{array}$ & $\begin{array}{l}\text { How do premedical } \\
\text { and former premedi- } \\
\text { cal students differ on } \\
\text { perceptions of a } \\
\text { medical career and } \\
\text { reasons for desiring a } \\
\text { medical career? How } \\
\text { does this differ by } \\
\text { years in school and by } \\
\text { gender? }\end{array}$ & $\begin{array}{l}\text { Survey/Cross- } \\
\text { sectional } \\
\text { using two } \\
\text { different } \\
\text { question- } \\
\text { naires. }\end{array}$ & $\begin{array}{l}\text { Self-reported } \\
\text { career aspirations } \\
\text { and reasons for } \\
\text { leaving the } \\
\text { premedical track }\end{array}$ & $\begin{array}{l}\text { Current } \\
\text { premedical } \\
\text { students and } \\
\text { former } \\
\text { premedical } \\
\text { students, } \\
\text { McDaniel } \\
\text { College }\end{array}$ & $\begin{array}{l}\text { Total sample } 97 \\
\text { students ( } 44 \text { former } \\
\text { premedical, } 53 \\
\text { current premedical) }\end{array}$ & $100 \%$ & $\begin{array}{l}\text { Limited generalizability } \\
\text { because convenience } \\
\text { sampling method, } \\
\text { small sample size, and } \\
\text { sampled from one, } \\
\text { small, private liberal } \\
\text { arts college; Upper- } \\
\text { classmen dispropor- } \\
\text { tionately represented in } \\
\text { both comparison } \\
\text { groups. }\end{array}$ \\
\hline
\end{tabular}

\begin{tabular}{|c|c|c|c|c|c|c|c|}
\hline $\begin{array}{l}\text { Manaster } \\
\text { et al } 1976^{35}\end{array}$ & $\begin{array}{l}\text { How do incoming } \\
\text { premedical students' } \\
\text { attitudes about } \\
\text { medicine compare to } \\
\text { those who are } \\
\text { currently applying to } \\
\text { medical school? How } \\
\text { does this differ by } \\
\text { gender? }\end{array}$ & $\begin{array}{l}\text { Survey/Cross- } \\
\text { sectional }\end{array}$ & $\begin{array}{l}\text { Self-reported } \\
\text { measures of social } \\
\text { background, } \\
\text { perceptions of } \\
\text { medicine and } \\
\text { medical school, } \\
\text { perception of } \\
\text { family relations, } \\
\text { parents, and self } \\
\text { during childhood, } \\
\text { personality items } \\
\text { that assess anxiety, } \\
\text { extraversion, and } \\
\text { internality }\end{array}$ & $\begin{array}{l}\text { Incoming } \\
\text { premedical } \\
\text { students and } \\
\text { premedical } \\
\text { students } \\
\text { applying to } \\
\text { medical school, } \\
\text { University of } \\
\text { Texas }\end{array}$ & $\begin{array}{l}\text { Total sample } 554 \\
\text { students ( } 372 \\
\text { incoming premeds, } \\
182 \text { premeds applying } \\
\text { to medical school) }\end{array}$ & $\begin{array}{l}\text { Potential } \\
\text { participants } \\
\text { approached - } \\
\text { not reported }\end{array}$ & $\begin{array}{l}\text { Limited generalizabil- } \\
\text { ity- sampled from one, } \\
\text { large university; } \\
\text { Skewed male/female } \\
\text { ratio; Limited detail } \\
\text { if/how questionnaire } \\
\text { was pretested; How } \\
\text { measured variables } \\
\text { related to impact of } \\
\text { collegiate experience } \\
\text { on occupational goals } \\
\text { not clearly defined. }\end{array}$ \\
\hline $\begin{array}{l}\text { McCranie } \\
\text { and Lewis } \\
1987^{36}\end{array}$ & $\begin{array}{l}\text { What is the preva- } \\
\text { lence of Type A } \\
\text { behavior among } \\
\text { premedical students } \\
\text { and students } \\
\text { pursuing other } \\
\text { courses of study? }\end{array}$ & $\begin{array}{l}\text { Survey/ Semi- } \\
\text { longitudinal }\end{array}$ & $\begin{array}{l}\text { Self-reported } \\
\text { measure of Type A } \\
\text { behavior, and } \\
\text { measures of } \\
\text { involvement with } \\
\text { premedical studies }\end{array}$ & $\begin{array}{l}\text { Premedical } \\
\text { students and } \\
\text { non premedical } \\
\text { students from } 13 \\
\text { private, liberal } \\
\text { arts colleges in } \\
\text { the Associated } \\
\text { Colleges of the } \\
\text { Midwest. }\end{array}$ & $\begin{array}{l}\text { Total sample } 253 \\
\text { students }(118 \\
\text { premed, } 73 \text { bio/chem } \\
\text { majors, } 62 \text { other } \\
\text { majors) }\end{array}$ & $\begin{array}{l}47.9 \% \text { of } \\
\text { initial sample } \\
\text { responded to } \\
\text { questionnaire; } \\
\text { Groupings } \\
\text { made to } \\
\text { resurvey a } \\
\text { subset of } 336 \\
\text { where } 75.3 \% \\
\text { responded. }\end{array}$ & $\begin{array}{l}\text { Limited generalizability } \\
\text { - low response rate, } \\
\text { sampled dispropor- } \\
\text { tionally from } \\
\text { predetermined groups } \\
\text { and sampled from } \\
\text { small private liberal } \\
\text { arts college; Selective } \\
\text { attrition }\end{array}$ \\
\hline
\end{tabular}


Table 2. Studies included in critical review (cont)

\begin{tabular}{|c|c|c|c|c|c|c|c|}
\hline Source & Research question & Method & Measures & Population & Sample size & $\begin{array}{l}\text { Response } \\
\text { rate }\end{array}$ & $\begin{array}{l}\text { Methodological } \\
\text { limitations }\end{array}$ \\
\hline $\begin{array}{l}\text { Pascarella } \\
\text { et al } 1987^{30}\end{array}$ & $\begin{array}{l}\text { What are the direct } \\
\text { and indirect effects of } \\
\text { undergraduate college } \\
\text { experience on } \\
\text { occupational } \\
\text { attainment in } \\
\text { medicine? }\end{array}$ & $\begin{array}{l}\text { Survey/ } \\
\text { Longitudinal- } \\
\text { Qualitative } \\
\text { causal } \\
\text { modeling }\end{array}$ & $\begin{array}{l}\text { Self-reported } \\
\text { measures of family } \\
\text { background, } \\
\text { secondary school } \\
\text { experiences, initial } \\
\text { occupational } \\
\text { aspirations, and } \\
\text { personal character- } \\
\text { istics }\end{array}$ & $\begin{array}{l}\text { National sample } \\
\text { of premedical } \\
\text { students, } \\
\text { enrolled in a } 4 \\
\text { year undergrad- } \\
\text { uate institution } \\
\text { in 1971, who } \\
\text { responded to } \\
\text { national CIRP } \\
\text { survey; followed } \\
\text { over } 9 \text { years. }\end{array}$ & $\begin{array}{l}\text { Total sample } 454 \\
\text { premedical students }\end{array}$ & $\begin{array}{l}\text { Secondary } \\
\text { data analysis } \\
(\mathrm{n} / \mathrm{a})\end{array}$ & $\begin{array}{l}\text { Selective attrition- } \\
\text { difficult to assess } \\
\text { effects of aspiration } \\
\text { changes with single } \\
\text { follow-up; Secondary } \\
\text { analysis of existing } \\
\text { data- survey not } \\
\text { designed around } \\
\text { research question and } \\
\text { weak operational } \\
\text { definitions model } \\
\text { variables. (i.e., the } 4 \\
\text { college experience } \\
\text { variables limited in } \\
\text { how they assess the } \\
\text { "experience" of the } \\
\text { student.) }\end{array}$ \\
\hline $\begin{array}{l}\text { Sade et al } \\
1984^{34}\end{array}$ & $\begin{array}{l}\text { Does the anecdotally } \\
\text { documented } \\
\text { "premedical } \\
\text { syndrome" exist in } \\
\text { reality and what traits } \\
\text { comprise is? }\end{array}$ & $\begin{array}{l}\text { Study 1: } \\
\text { Survey/Cross- } \\
\text { sectional; } \\
\text { Study 2: } \\
\text { Survey/Cross- } \\
\text { sectional }\end{array}$ & $\begin{array}{l}\text { Self-reported } \\
\text { ratings of } \\
\text { premedical } \\
\text { students on } 14 \\
\text { different traits, and } \\
\text { self-reported major } \\
\text { of study }\end{array}$ & $\begin{array}{l}\text { Study 1: } \\
\text { Premedical and } \\
\text { non-premedical } \\
\text { students from } 13 \\
\text { colleges in South } \\
\text { Carolina; Study } \\
\text { 2: Faculty } \\
\text { members } 13 \\
\text { colleges in South } \\
\text { Carolina }\end{array}$ & $\begin{array}{l}\text { Study 1: total sample } \\
498 \text { students; ( } 253 \\
\text { premed, } 245 \\
\text { randomly sampled } \\
\text { non-premed); Study } \\
\text { 2: } 403 \text { faculty }\end{array}$ & $\begin{array}{l}\text { Study } 1 \& 2 \text { : } \\
\text { Potential } \\
\text { participants } \\
\text { approached - } \\
\text { not reported }\end{array}$ & $\begin{array}{l}\text { Limited generalizabil- } \\
\text { ity- sampled, small, } \\
\text { colleges in one } \\
\text { southern state; Data are } \\
\text { limited to perceptions } \\
\text { and not direct } \\
\text { observations; Limited } \\
\text { information on items } \\
\text { in questionnaire and if } \\
\text { it was pretested. }\end{array}$ \\
\hline $\begin{array}{l}\text { Simmons } \\
2005^{37}\end{array}$ & $\begin{array}{l}\text { What are the } \\
\text { attitudes of premedi- } \\
\text { cal students towards } \\
\text { breadth of education? }\end{array}$ & $\begin{array}{l}\text { Study 1: } \\
\text { Interviews; } \\
\text { Study 2: Focus } \\
\text { group } \\
\text { interviews } \\
\text { with subset of } \\
\text { Study 1 } \\
\text { participants }\end{array}$ & $\begin{array}{l}\text { Interviews and } \\
\text { focus groups } \\
\text { focused on student } \\
\text { attitudes towards } \\
\text { educational } \\
\text { breadth }\end{array}$ & $\begin{array}{l}\text { Study 1: } \\
\text { Premedical } \\
\text { students, junior } \\
\text { and senior years, } \\
\text { Centerville } \\
\text { University }\end{array}$ & $\begin{array}{l}\text { Study 1:15 students; } \\
\text { Study 2: } 7 \text { students }\end{array}$ & $\begin{array}{l}\text { Study } 1: 23 \text { of } \\
1100(.02 \%) \\
\text { students } \\
\text { responded to } \\
\text { initial } \\
\text { solicitation; } 16 \\
(69 \%) \\
\text { completed } \\
\text { interview and } \\
1 \text { eliminated } \\
\text { based on } \\
\text { study criteria; } \\
\text { Study 2: Not } \\
\text { reported. }\end{array}$ & $\begin{array}{l}\text { Limited generalizabil- } \\
\text { ity- sampled one } \\
\text { university, focused on } \\
\text { liberal arts education; } \\
\text { Self-selection bias due } \\
\text { to small sample size } \\
\text { drawn from large web } \\
\text { recruitment effort; } \\
\text { Selection bias due to } \\
\text { purposeful sampling } \\
\text { technique; Selection } \\
\text { bias towards students } \\
\text { who remain in the } \\
\text { premed program } \\
\text { because sample of } \\
\text { upper classmen; } \\
\text { Skewed male/female } \\
\text { ratio. }\end{array}$ \\
\hline $\begin{array}{l}\text { Staley and } \\
\text { Hood } \\
1977^{22}\end{array}$ & $\begin{array}{l}\text { What are the causes } \\
\text { of higher attrition } \\
\text { rates for women in } \\
\text { premedical pro- } \\
\text { grams? }\end{array}$ & $\begin{array}{l}\text { Longitudinal } \\
\text { survey }\end{array}$ & $\begin{array}{l}\text { Self-reported } \\
\text { measures of } \\
\text { student family } \\
\text { characteristics, } \\
\text { personal and } \\
\text { educational } \\
\text { background, and } \\
\text { past and future } \\
\text { medical career } \\
\text { plans }\end{array}$ & $\begin{array}{l}\text { Premedical } \\
\text { students, in their } \\
\text { freshman and } \\
\text { sophomore } \\
\text { years, University } \\
\text { of Iowa }\end{array}$ & $\begin{array}{l}\text { Total sample } 188 \\
\text { students ( } 99 \text { female, } \\
89 \text { male) }\end{array}$ & $\begin{array}{l}\text { Initial } \\
\text { response rate } \\
\text { to survey } \\
\text { invitation not } \\
\text { reported; } \\
\text { Sample } \\
\text { population at } \\
\text { T1:89\% of } \\
\text { females (106); } \\
81 \% \text { of males } \\
\text { (98); Sample } \\
\text { population at } \\
\text { T2: 93\% } \\
\text { females (99); } \\
91 \% \text { males } \\
\text { (89) }\end{array}$ & $\begin{array}{l}\text { Limited generalizabil- } \\
\text { ity- sampled one, large } \\
\text { Midwestern university; } \\
\text { Selective attrition; } \\
\text { Sampling times exclude } \\
\text { measurement of } \\
\text { changes to perceptions } \\
\text { over time after } \\
\text { sophomore year; } \\
\text { Limited information } \\
\text { on items in question- } \\
\text { naire and if it was } \\
\text { pretested. }\end{array}$ \\
\hline
\end{tabular}


reality of a medical career. ${ }^{25,26}$ These studies, done more than 20 years ago, indicated that those who left the premedical track often did so as a result of a change in individual-level goals or aspirations.

By contrast, more recent work examined the ways premedical students are pushed out of medical careers by negative experiences with required coursework. These studies focus on the attrition of under-represented minorities from the premedical track, demonstrating that negative experiences with required chemistry courses - especially for women from under-represented minority groups drove students out of the premedical track..$^{27-29}$ Unlike those described in earlier studies, these students remained interested in medicine, but left the premedical track because they believed they could not survive medical school courses. These more recent studies saw attrition from the premedical track, especially on the part of women and under-represented minorities, as the result of structural factors, rather than lack of interest or motivation on the part of the students.

Three separate articles offered a slightly different perspective on student survival during the premedical years. Pascarella and colleagues found that the quality of the undergraduate institution influenced success in gaining admission to medical school. ${ }^{30}$ While most other articles focused on premedical students in general, this article examined how different undergraduate institutions influenced the students' progress from the premedical to medical stage. Klink et al., documented the importance of a premedical student's support network for survival through the premedical years: students with better familial support were more likely to believe in their own coping efficacy. ${ }^{31}$ Finally, Chuck surveyed premedical students and found that most were excessively idealistic about the daily work of a physician, when compared with practicing physicians, suggesting that an idealistic view of medicine may contribute to increasing numbers of medical school applications. ${ }^{32}$ With regards to idealism, it is interesting to note that several studies found that many premedical students report a desire to help others as both a reason to pursue medicine, ${ }^{32,33}$ as well as a reason to leave the premedical track when they come to believe that their desire to help others can no longer be fulfilled by pursuing a career in medicine. ${ }^{25,26,29}$

The premedical personality-stereotypes and the "premedical syndrome"

The remaining eight of the nineteen articles examined the personality traits of premedical students, exploring the concept of a premedical stereotype or "syndrome." Studies done in the 1970s and early 1980s depicted the personality traits of premedical students negatively. These studies reported that premedical students were less social and more concerned with money and prestige than other students, claiming that both non-premedical and premedi- cal students saw premedical students as overly competitive, excessively grade-conscious overachievers, with narrow academic interests. ${ }^{33,34}$ This body of research also found that premedical students concentrated primarily in the sciences, rather than diversifying their academic profiles. Manaster and colleagues found this mentality to be more prevalent during the earlier half of the premedical years. Once premedical students applied to medical school, according to this study, they matured and became more self-assured, demonstrating less of these stereotypical qualities. $^{35}$

Studies published in the mid-1980s to 2010 discovered that the premedical stereotype was more a perception than an observed reality. These studies found that while premedical students were competitive and cared a great deal about grades, they were no different in these respects from other students planning for graduate study in the biological or physical sciences. ${ }^{36,37}$ Furthermore, unlike previous studies that characterized premedical students as narrowly focused, these studies found that premedical students valued enrolling in a broad range of courses - to a greater extent than other biology or chemistry majors - and cared about mastering course content, rather than working exclusively for the grade. ${ }^{38,39}$ Conrad found that while many premedical students recognized the stereotype of premedical students as cutthroat and competitive, most premedical students did not actually exhibit cutthroat or competitive behavior. ${ }^{40}$ Instead, many participated in cooperative efforts with their classmates. According to Conrad, the premedical stereotype was a construct that premedical students used to understand their failures and successes within the context of an extremely competitive academic environment.

Taken together, these studies demonstrated that premedical students' behaviors and motivations are more complicated than they appear at first glance. Those who choose to pursue medicine often balance the necessity of maintaining competitive grades in rigorous coursework against a desire to take a wide variety of classes and develop mastery over course content.

\section{Limitations of existing research}

There are several limitations to the research we reviewed. Most striking is the paucity of empirical studies of the premedical students in the US and the dated nature of the research. Of the nineteen studies included in the review, only ten were published after 1990. Many questions about the premedical experience remain unanswered and at most, the existing literature provides a basis for comparison to today's students.

Additionally, differences in sampling strategies across studies make it particularly difficult to draw general conclusions about premedical students. It is worth noting that the premedical student population is particularly challenging to identify and sample, since anything prior to 
medical school could be considered "premedical." In fact, studies used a variety of definitions of a premedical student to identify their samples of interest. Some studies used the Association of American Medical Colleges (AAMC) data on the MCAT to identify their samples. ${ }^{25,26}$ Others used enrollment in premedical-required courses as indicators. $^{23,24,31}$ Those authors with connections to the premedical advising structure used email lists and social networks to recruit participants. ${ }^{34,37,40}$ The most common sampling

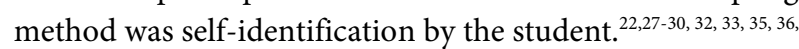
${ }^{38,39}$ Each of these methods yielded slightly different samples of premedical students, which, in turn, can influence the conclusions drawn.

Future studies should attempt to sample all types of premedical students, including those who eventually leave the premedical track. Furthermore, if we are to understand how premedical students identify themselves and the factors that influence attrition from the premedical track we must also follow samples of premedical students through their college careers, observing how bright-eyed and eager first- year premedical students are shaped by their college experiences.

\section{Discussion}

While based on sparse information, our review demonstrates that research on premedical students has focused primarily on two questions: why do students leave the premedical track and what are the attributes and personality characteristics of a premedical student? Research suggests that in the 1970s through the 1980s attrition was linked to students' decreased desire to pursue a medical career. More recent studies found that negative experiences with coursework, and particularly with chemistry classes, led students to leave the premedical track. The premedical stereotype - a largely negative image of premedical students as single-minded, competitive, and obsessed with grades - continues to have currency inside and outside the premedical world, in spite of research that shows that premeds are, in fact, a diverse group of students with a broad range of interests.

The studies reviewed suggest that the premedical experience may be difficult and overly competitive, leading some would-be doctors to seek other careers. According to this research, the premedical experience is shaped by the participation of high-achieving, goal-oriented - and in some instances, overtly competitive-premedical students in challenging curricular requirements, creating an environment that may ultimately dissuade some students from applying to medical school. For students with unrealistic expectations of careers in medicine, participation in such an environment may result in an appropriate decision to change careers. However, it is also possible that some capable and dedicated students who are not ready for the rigorous demands of premedical coursework may be pushed into an inappropriate early decision to abandon their career aspirations. Thus these studies highlight the potential role of the premedical experience in pushing potentially capable doctors out of the premedical track.

\section{Conclusions}

Our critical review of the literature underscores the need for up-to-date, high quality empirical research on the premedical experience. Some of the research reviewed here was published nearly 40 years ago. Over the past four decades medicine has seen dramatic changes: increasing numbers of women and underrepresented minorities entering the profession and dramatic shifts in the financing and organization of healthcare that have altered the requirements for, and the experience of, premedical and medical education.41 Studies of premedical students conducted many years ago likely have little application to current premedical education experiences, particularly for women and underrepresented minorities. Additionally, medical education itself has changed, becoming broader and more interdisciplinary, emphasizing the social determinants of health, doctor-patient communication and bioethics, in addition to anatomy and physiology. One need look no further than the recent changes to the MCAT, with the addition of two new test sections emphasizing the social sciences, to see the changes undergoing the medical education system. ${ }^{5}$ We need updated research to examine how the premedical experience in this new context of medical education is shaping the physicians of tomorrow.

We also need more empirical research in medical education to focus on the premedical years. To our knowledge, our review is the first to critically synthesize information from the empirical literature on premedical students and we found few articles that met our search criteria. Though premedical education is a topic about which much has been written, most of that writing has been in either in the form of opinion pieces or published in national reports of various professional organizations. ${ }^{42}$ Thus, the few articles that met our search criteria is an important and also serendipitous finding of our review, confirming our larger point that we have much to learn about the how the premedical years shape the physician workforce.

Interestingly, our review also identifies a shift in the conceptualization of the premedical student and the premedical experience over time. Earlier studies of premedical students focused on the individuals who choose to pursue premedical studies at the undergraduate level. These studies show premedical students to be overly ambitious, to the point of being perceived as cutthroat, or likely to leave premedical studies because of a growing disaffection with medicine. These studies focused on the individual-the premedical experience is described as a function of individual personality traits or individual aspirations, with little attention paid to the contextual influences on premedical students. More recent literature, 
however, recognizes the importance of context for the premedical experience. These studies note that the premedical experience is shaped, not just by personal characteristics, but also by formal curricular requirements and strong social norms that influence the identity of an ideal and successful premed student. While we lack the data to explain this shift in research focus, this change may be the result of the increasing influence of medical sociology on studies of medical education, as well as increasing attention to the influences of the hidden curriculum on the part of medical educators. ${ }^{43,44}$

On a related note, it is interesting to note that research has primarily focused on two topics - attrition and the premedical stereotype-leaving much room for other research questions regarding how the premedical experience influences those who wish to become doctors. One topic that deserves special attention is the hidden curriculum of the premedical years. We need studies designed to understand the overall culture of premedical education and not just the traits of individual premedical students. While there is a significant body of literature demonstrating the existence of the rich subcultures that emerge among both medical students and residents in response to stresses in the environment, ${ }^{45-47}$ we were unable to find similar studies at the undergraduate level. Therefore, we know little about premedical culture-such as the survival strategies students collectively develop during this period - and the effects of that culture on future physicians. Rather than continue to focus disproportionately on the academic content of the formal premedical curriculum, new research should pay attention to the informal and hidden curricula-the tacit knowledge premedical students learn informally from advisors, parents, and peers-from watching what they say as well as what they do. ${ }^{16}$

In short, our review demonstrates a need for more high-quality and updated research on the premedical years, and in particular research that focuses on the premedical experience. We know more about the personal characteristics of premedical students than we do about the premedical subculture. We know more about the formal curriculum than about the hidden curriculum. Furthermore, we know little about those who are not admitted and still less about those who abandon their premedical aspirations. According to the AAMC, there were 42,742 applicants for approximately 19,000 seats in medical school in $2010 .^{48}$ This group, however, was drawn from an even larger, unknown number of college undergraduates who participated in the undergraduate premedical experience but did not apply to medical school. If we wish to influence the character of future physicians we must pay attention to what happens to students on their way to medical school. We must explore how this period influences students' ideas about success, relationships, and caring for others. ${ }^{15}$ In order to gain a deeper and more complete understanding of the physicians of the future, researchers must give equal attention to the first, critical steps occurring during the professionalization process: the premedical years.

\section{Acknowledgments}

We thank Whitney Townsend, the coordinator for the Health Sciences Executive Research Services, for her assistance on conducting our database searches. We would like to thank Erica Blom for her research assistance in the initial stages of the search. Ms. Lin is supported in part by a grant from the Office of the Vice President of Research and an NIA training grant to the Population Studies Center at the University of Michigan (T32 AG000221), University of Michigan, Mr. Crawford by the Center for Ethics in Public Life, University of Michigan and Dr. De Vries is supported in part by a grant from the National Library of Medicine (1G13LM008781-01).

\section{Conflict of Interest}

The authors declare that they have no conflict of interest.

\section{References}

1. Irby DM, Cooke M, O'Brien BC. Calls for reform of medical education by the Carnegie Foundation for the Advancement of Teaching: 1910 and 2010. Acad Med. 2010;85(2):220-7.

2. Gunderman RB, Kanter SL. Perspective: "how to fix the premedical curriculum" revisited. Acad Med. 2008;83(12):1158-61.

3. Emanuel EJ. Changing premed requirements and the medical curriculum. JAMA. 2006;296(9):1128-31.

4. Dienstag JL. Relevance and rigor in premedical education. N Engl J Med. 2008;359(3):221-4.

5. Kaplan RM, Satterfield JM, Kington RS. Building a better physician -the case for the new MCAT. N Engl J Med. 2012;366:1265-8.

6. James D, Chilvers C. Academic and non-academic predictors of success on the Nottingham undergraduate medical course 1970-1995. Med Educ. 2001;35(11):1056-64.

7. Yates J, James D. Predicting the "strugglers": a case-control study of students at Nottingham university medical school. BMJ. 2006;332(7548) :1009-13.

8. Forester JP, McWhorter DL, Cole MS. The relationship between premedical coursework in gross anatomy and histology and medical school performance in gross anatomy and histology. Clin Anat. 2002;15(2):160-4. 9. Stewart SM, Lam TH, Betson CL, Wong CM, Wong AM. A prospective analysis of stress and academic performance in the first two years of medical school. Med Educ. 1999;33(4):243-50.

10. Hafferty FW, Franks R. The hidden curriculum, ethics teaching, and the structure of medical education. Acad Med. 1994;69(11):861-71.

11. Culver CM, Clouser KD, Gert B, Brody H, Fletcher J, Jonsen A, et al. Basic curricular goals in medical ethics. N Engl J Med. 1985;312(4):253-6.

12. Thomas NK. Resident burnout. JAMA. 2004;292(23):2880-9.

13. Strous RD, Shoenfeld N, Lehman A, Wolf A, Snyder L, Barzilai O. Medical students' self-report of mental health conditions. Int J Med Educ. 2012;3:1-5.

14. Schwenk TL, Davis L, Wilmsatt LA. Depression, stigma, and suicidal ideation in medical students. JAMA. 2010;304(11):1181-90.

15. Gross JP, Mommaerts CD, Earl D, De Vries RG. After a century of criticizing premedical education, are we missing the point? Acad Med. 2008;83(5):516-20.

16. Hafferty FW. Beyond curriculum reform: confronting medicine's hidden curriculum. Acad Med. 1998;73(4):403-7.

17. Salvatori P. Reliability and validity of admissions tools used to select students for the health professions. Adv Health Sci Educ Theory Pract. 2001;6(2):159-75.

18. Ferguson E, James D, Madeley L. Factors associated with success in medical school: systematic review of the literature. BMJ. 2002;324(7343):952-7. 
19. Eva KW. On the limits of systematicity. Med Educ. 2008;42(9):852-3. 20. Matriculating student questionnaire: 2011 all schools summary report. Washington, DC: American Association of Medical Colleges; 2011 [cited 31 March 2012]; Available from: https://www.aamc.org/download/269322/ data/msq2011.pdf.

21. Olson SW. Combined-degree programs: a valuable alternative for motivated students who choose medicine early. Acad Med. 1992;67(11) :783-4.

22. Staley $\mathrm{KH}$, Hood AB. A longitudinal study of female premedical attrition. J Med Educ. 1977;52(10):849-51.

23. Fiorentine R. Men, women, and the premed persistence gap: a normative alternatives approach. AJS. 1987;92(5):1118-39.

24. Fiorentine R, Cole S. Why fewer women become physicians: explaining the premed persistence gap. Sociol Forum. 1992;7(3):469-96.

25. Colquitt WL, Killian CD. Students who consider medicine but decide against it. Acad Med. 1991;66(5):273-8.

26. Barondess JA, Glaser RJ. Attitudes toward the medical career: findings from the Alpha Omega Alpha survey of college and university undergraduates. Acad Med. 1993;68(5):323-8.

27. Barr DA, Gonzalez ME, Wanat SF. The leaky pipeline: factors associated with early decline in interest in premedical studies among underrepresented minority undergraduate students. Acad Med. 2008;83(5):503-11.

28. Barr DA, Matsui J, Wanat SF, Gonzalez ME. Chemistry courses as the turning point for premedical students. Adv Health Sci Educ Theory Pract. 2010;15(1):45-54.

29. Lovecchio K, Dundes L. Premed survival: understanding the culling process in premedical undergraduate education. Acad Med. 2002;77 (7):719-24.

30. Pascarella ET, Brier EM, Smart JC, Herzog L. Becoming a physician: the influence of the undergraduate experience. Res in High Educ. 1987;26(2):180-201.

31. Klink JL, Byars-Winston A, Bakken LL. Coping efficacy and perceived family support: potential factors for reducing stress in premedical students. Med Educ. 2008;42(6):572-9.

32. Chuck JM. Do premedical students know what they are getting into? West J Med. 1996;164(3):228-30.

33. Hackman JD, Low-Beer JR, Wugmeister S, Wilhelm RC, Rosenbaum JE. The premed stereotype. J Med Educ. 1979;54(4):308-13.

34. Sade RM, Fleming GA, Ross GR. A survey on the 'premedical syndrome'. J Med Educ. 1984;59(5):386-91.

35. Manaster GJ, Friedman ST, Larson D. Premedical students survivability and specialization: a social and psychological study. Psychol Rep. 1976;39(1):35-45.

36. McCranie EW, Lewis GL. Prevalence of type A behavior among undergraduate students with medical and non-medical career plans. J Med Educ. 1987;62(11):926-8.

37. Simmons A. Beyond the premedical syndrome: premedical student attitudes toward liberal education and implications for advising. NACADA Journal. 2005;25(1):64-73.

38. Lewis GL. Course work and grades of premedical students at two liberal arts colleges. J Med Educ. 1985;60(9):677-83.

39. Horowitz G. It's not always about the grade: exploring the achievement goal orientations of pre-med students. J Exp Educ. 2010;78(2):215-45.

40. Conrad P. The myth of the cut-throats among premedical students: on the role of stereotypes in justifying failure and success. J Health Soc Behav. 1986;27(2):150-60.

41. Anspach RR. Gender and health care. In: Bird CE, Conrad P, Fremont AM, Timmermans S, editors. Handbook of Medical Sociology, 6th edition. Nashville, TN: Vanderbilt University Press; 2010. p. 229-48.

42. Physicians for the twenty-first century--the GPEP report: report of the panel on the general professional education of the physician and college preparation for medicine. Washington, DC: Association of American Medical Colleges. 1984.

43. Hafferty FW, Castellani B. The hidden curriculum: a theory of medical education. In: Brosnan C, Turner BS, editors. Handbook of the Sociology of Medical Education. New York, New York: Routledge; 2009. p. 15-35.

44. Stern DT, Papadakis M. The developing physician - becoming a professional. N Engl J Med. 2006;355(17):1794-9.

45. Bosk CL. Forgive and remember: managing medical failure (2nd ed). Chicago, IL: The University of Chicago Press; 2003.

46. Becker HS, Geer B, Hughes EC, Strauss AL. Boys in white: student culture in medical school. Chicago, IL: The University of Chicago Press; 1961.

47. Mizrahi T. Managing medical mistakes: ideology, insularity and acco untability among internists-in-training. Soc Sci Med. 1984;19(2):135-46.

48. U.S. Medical School Applicants and Students 1982-1983 to 2010-2011. Washington, DC: American Association of Medical Colleges; 2010 [cited 21 February 2011]; Available from: https://www.aamc.org/download/ 153708/data/charts1982to2012.pdf.

Appendix I: Databases searched and search strategies

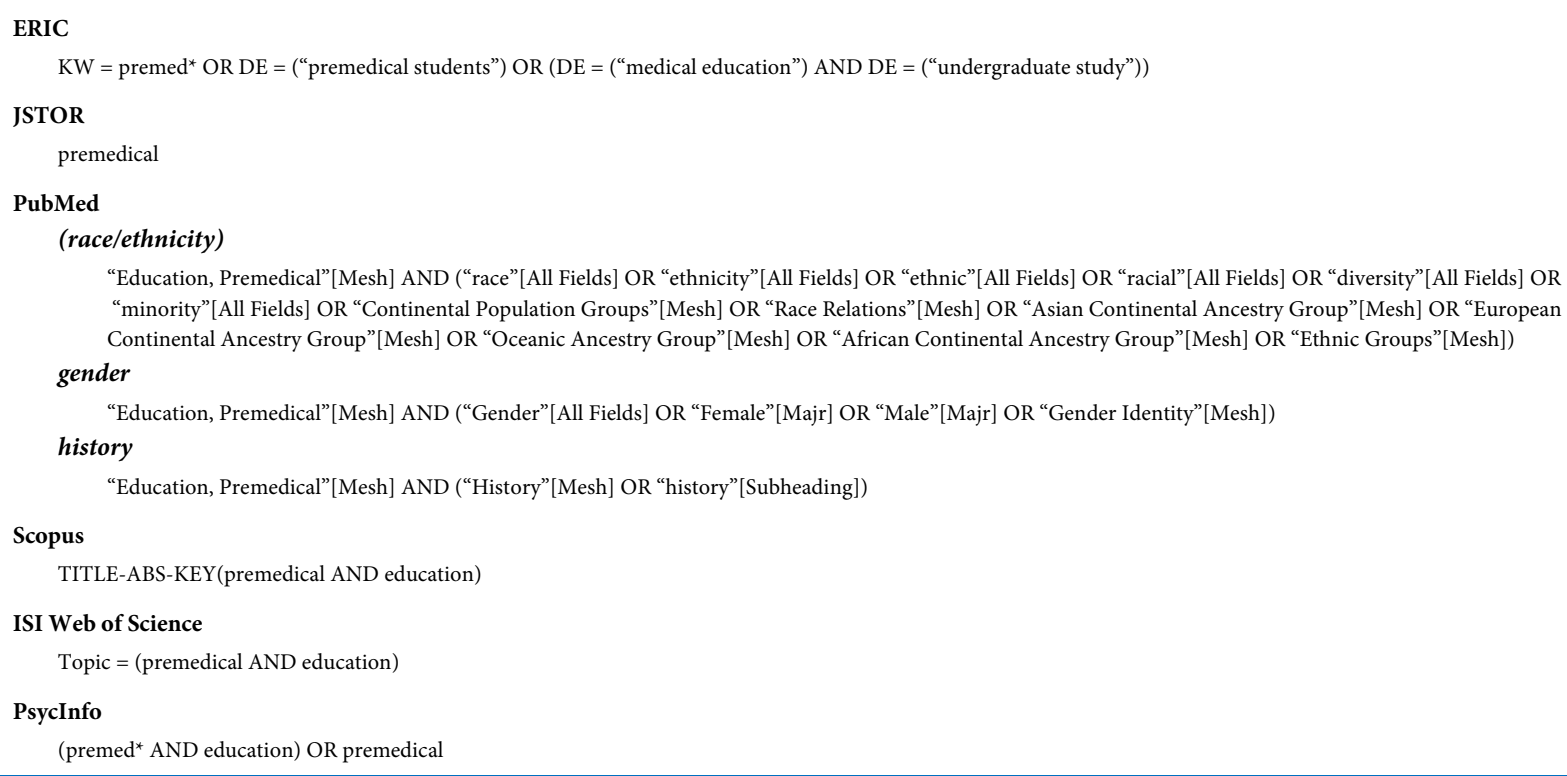

